
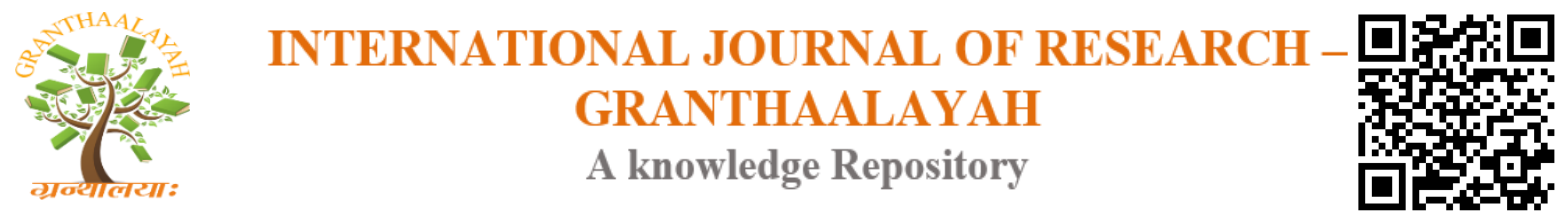

Social

\title{
ENVIRONMENTAL MANAGEMENT IN THE HINTERLANDS OF NORTHERN SAMAR
}

\author{
Felisa L. Sanico *1 \\ ${ }^{* 1} \mathrm{PhD}$, Vice President for Academic Affairs, Author
}

\begin{abstract}
This study investigated the perceptions of the faculty of members, school official and students in terms of environmental protection and management. It employed random sampling using survey questionnaires to gather data from the respondents. Frequency counts and percentage were used to process the data.
\end{abstract}

Most of the respondents had positive responses on waste reduction and management, as the commonly used environmental protection strategy in the school. A majority of them rejected plastics and agreed that these are harmful to the environment friendly products, are materials that can be recycled, and less favored materials are the non-toxic products, and a majority of the respondents favored that individual bulk packaging is preferred than bulk packaging of products from suppliers.

High percentage favored that the classrooms are designed to make use of natural ventilation and natural lighting. Most of the respondents had negative responses on the issue of planning the campus in such a way as to reduce the use of motor vehicle in terms of motor boat as mode of transportation. There was a high percentage of positive response regarding the physical greening of the school, as observed in the entire campus. A majority of the respondents had positive responses on fluorescent lamps as the commonly used source of light, than of the use of incandescent. Most of the respondents had negative response on artesian well as the source of water, and a high positive response was observed regarding local water works as the source of water for everyday use. Majority of the respondents agreed that office machines are turned off when not in use, and using the fans whenever possible also had a high positive response; only fewer respondents agreed on the usage of air conditioner. A majority of the respondents agreed that white paper is the most preferred kind of paper in the school.

A majority of the respondents had positive response in using the used paper in photocopying articles as commonly used paper recycling or use of reduction program. A majority of the respondents agreed that composting biodegradable was right in the campus and wet waste sent to recyclers had a low positive response. There was a high percentage of positive response on disposable plastic utensils as the most discouraged material in the canteen. Disposable cups, disposable plates, paper napkins and plastic wrappers had also high percentage as materials discouraged in the canteen. A majority of the respondents agreed that waste segregation in the school is practiced. Most of the respondents agreed that containers for biodegradable and nonbiodegradable are provided in the entire campus. A majority of the respondents favored that 
biodegradable materials are made to compose. Respondents favored that sealing properly before disposal of hazardous/chemicals water is done inside the campus.

All the eight (8) environment concerns/themes are integrated in the curriculum. A majority of the respondents indicated that environment concerns/themes are incorporated in Music, Health and Physical Education. They are also integrated in English, Social Studies, Pilipino, and Mathematics.

Keywords: Environmental Management; Environmental Policy; Environmental Education; Waste Management; Environmental Protection and Management Strategies.

Cite This Article: Felisa L. Sanico. (2019). "ENVIRONMENTAL MANAGEMENT IN THE HINTERLANDS OF NORTHERN SAMAR." International Journal of Research - Granthaalayah, 7(3), 163-176. https://doi.org/10.29121/granthaalayah.v7.i3.2019.957.

\section{Introduction}

Education is the main avenue through which society prepares its citizens in carrying out their responsibility (Environmental Education Guide, 1990).

Environmental education is one of the factors that can help protect the environment. As Silvino Lobos is an instrument that produce graduates that are not only globally competitive but also environmentally literate, the level of implementation of the schools on environmental protection, including strategies, and awareness in the process of educating the students need to be investigated, thus this study.

The extent of environmental destruction in the Philippines in general is alarming. The 1988 Haribon-generated image (based on NAMRIA, 1988 references) and the TREES-generated image of the deforestation rate in the Philippines highlights the extent of deforestation in the Visayas. The area, thus, is an identified site of endangered species because of its threatened ecosystem.

Northern Samar is one of the Visayan provinces that boosts of rich natural reseources, and these too are threatened. The waters surrounding the province host abundant marine resources-but droves of small and commercial fishers are threatening the sustainability of sea resources owing to over-and irresponsible fishing. Even at the present state of exploitation and open access fishing, the province has high fish yields.

The present state of the environment in Northern Samar reveals the fragile state of its ecosystems due to overuse and the demands of a growing population, abuse, unregulated human activities in all ecosystems, lack of concern for the environment, and weak political will of local government officials, among others. Environmental programs of local government units and agencies and even NGOs and the academe depend mostly on external funding. There is a need for the academe and local government units to explore innovative explorations into environemntal management, protection and conservation that draw from the social capital, passion and local resources of communities. Northern Samar, though productive, will drive its resources to the point of degradation if unregulated and unchecked. 
Environmental management should be incorporated in the school's educational goals because the resolution to environmental problems call active and conscious response from the students who are environmental literates. Thus, the researcher was prompted to conduct this study in order to determine how far the Silvino Lobos Vocational High School has gone into implementing environmental education, considering that it is one of the remotest towns, if not the remotest town in the Province of Northern Samar.

\section{Methodology}

The Municipality of Silvino Lobos is composed of 26 barangays with total area of 22,420 hectares. Silvino Lobos is a fourth class municipality, and according to the 2010 census, it has a population of 14,303. Its primary products are copra and abaca. Republic Act No. 7094 created the Silvino Lobos Vocational High School (SLVHS) and was signed into law in August 2, 1991, but it was started on August 2, 1993. The school is comprised of 6 buildings, with total land area of approximately 3.5 hectares. It is located in Poblacion 2 which has the biggest population among the three poblacion barangays in terms of high education and lies at the center of the municipality. It is consists of 102 households with 728 total population.

This study used the descriptive research design. It aimed to describe the perceptions of the Silvino Lobos Vocational High School particularly the teachers, school official, and students towards environmental protection and management. A survey questionnaire was used to gather the necessary data from the respondents. It was patterned from the Environmental Education Guide (Green Audit Form, 1999) which is designed by simply checking the box before the questions, for the respondents.

\section{The Respondents}

The respondents included 69 fourth year students, 14 teachers, and one (1) school official of the Silvino Lobos Vocational High School.

The teachers and school official were completely enumerated. The student respondents, on the other hand, were randomly selected through the fish-bowl method. In obtaining the sample size of student-respondents the formula below was used.

$$
\begin{array}{cc}
\boldsymbol{S}=\boldsymbol{N} & \\
\hdashline \boldsymbol{l}+\boldsymbol{N}(\boldsymbol{e})^{2} & \\
& \text { Where: } \mathbf{S}=\text { sample size } \\
& \mathbf{N}=\text { number of population } \\
& \mathbf{E}=0.05 \text { margin of error } \\
& \mathbf{1}=\text { constant }
\end{array}
$$

Actual survey was conducted to gather the needed data. Questionnaires were distributed to the respondents which were retrieved after they were answered. The data retrieved were recorded, tabulated and analyzed. The responses were tabulated and analyzed using frequency counts and percentages. The data are presented in tables. 


\section{Results and Discussion}

Silvino Lobos does not have a general environmental policy, but has a plan to formulate an environmental policy to be implemented next calendar year. However, environmental protection is part of the school even if there is no defined general environmental policy. The environmental consideration included in the criteria for decision making is to maintain the cleanliness of the school. Furthermore, there is no committee responsible for environmental projects and programs in the school. Environmental consideration is not included in the performance appraisal system of faculty, department head or school official and support staff or employees since the school does not have general environmental policy.

\subsection{Building and Grounds}

Table 1: Responses of Faculty Members, School Official, and Students on Structures Designed to Make Maximum Use of Natural Lighting and Ventilation

\begin{tabular}{|c|c|c|c|c|c|c|c|c|c|c|}
\hline \multirow{3}{*}{ Structure } & \multicolumn{3}{|c|}{ Students } & \multicolumn{3}{|c|}{ Faculty Members } & \multicolumn{3}{|c|}{ School Official } & \multirow[b]{3}{*}{ RANK } \\
\hline & $\mathbf{P}$ & $\mathbf{N}$ & NR & $\mathbf{P}$ & $\mathbf{N}$ & NR & $\mathbf{P}$ & $\mathbf{N}$ & NR & \\
\hline & $\begin{array}{c}\mathbf{f} \\
(\%)\end{array}$ & $\begin{array}{c}f \\
(\%)\end{array}$ & $\begin{array}{c}\mathbf{f} \\
(\%)\end{array}$ & $\begin{array}{c}\mathbf{F} \\
(\%)\end{array}$ & $\begin{array}{c}\mathbf{f} \\
(\%)\end{array}$ & $\begin{array}{c}\mathbf{f} \\
(\%)\end{array}$ & $\begin{array}{c}f \\
(\%)\end{array}$ & $\begin{array}{c}f \\
(\%)\end{array}$ & $\begin{array}{c}\mathbf{f} \\
(\%)\end{array}$ & \\
\hline Classroom & $\begin{array}{c}29 \\
(34.93) \\
\end{array}$ & $\begin{array}{c}14 \\
(16.86) \\
\end{array}$ & $\begin{array}{c}26 \\
(31.33) \\
\end{array}$ & $\begin{array}{c}11 \\
(13.25) \\
\end{array}$ & $\begin{array}{c}1 \\
(1.2) \\
\end{array}$ & $\begin{array}{c}2 \\
(2.4) \\
\end{array}$ & $\begin{array}{c}1 \\
(1.2) \\
\end{array}$ & 0 & 0 & 1 \\
\hline Canteen & $\begin{array}{c}8 \\
(9.6)\end{array}$ & $\begin{array}{c}24 \\
(28.9)\end{array}$ & $\begin{array}{c}37 \\
(44.58)\end{array}$ & $\begin{array}{c}2 \\
(2.4)\end{array}$ & 0 & $\begin{array}{c}12 \\
(12.46)\end{array}$ & 0 & 0 & 0 & 4 \\
\hline Gym & $\begin{array}{c}11 \\
(13.25)\end{array}$ & $\begin{array}{c}21 \\
(25.3)\end{array}$ & $\begin{array}{c}37 \\
(44.58)\end{array}$ & 0 & $\begin{array}{c}2 \\
(2.4)\end{array}$ & $\begin{array}{c}12 \\
(12.46)\end{array}$ & 0 & 0 & 0 & 3 \\
\hline Library & $\begin{array}{c}20 \\
(24.09)\end{array}$ & $\begin{array}{c}14 \\
(16.86)\end{array}$ & $\begin{array}{c}35 \\
(42.17)\end{array}$ & $\begin{array}{c}1 \\
(1.2)\end{array}$ & $\begin{array}{c}1 \\
(1.2)\end{array}$ & $\begin{array}{c}12 \\
(12.46)\end{array}$ & 0 & 0 & 0 & 2 \\
\hline TOTAL & $\begin{array}{c}68 \\
(81.93) \\
\end{array}$ & $\begin{array}{c}73 \\
(87.95) \\
\end{array}$ & $\begin{array}{c}135 \\
(162.7) \\
\end{array}$ & $\begin{array}{c}14 \\
(16.86) \\
\end{array}$ & $\begin{array}{c}4 \\
(4.8) \\
\end{array}$ & $\begin{array}{c}38 \\
(45.78) \\
\end{array}$ & $\begin{array}{c}1 \\
(1.2) \\
\end{array}$ & 0 & 0 & \\
\hline
\end{tabular}

On the responses on the school designed to make use of natural lighting, classroom ranked the highest as to structure designed to make use of natural lightning. This was followed the library from with 21 or $25.3 \%$ respondents in favor, $24.09 \%$ from the students, $1.2 \%$ from the faculty members, and no response from the school official. The gym ranked third with 11 or $13.25 \%$ from the students; however the faculty members and school official had no response. The canteen ranked the lowest with 8 or $9.6 \%$ from the students, $2.4 \%$ from the faculty members and no response from the school official. This only indicates that classrooms and library structures must be given importance in terms of maximum utilization of natural lighting considering their use and the number of population using. Daylighting has been touted for its many aesthetic and health benefits by designers and researchers. Scientists at the Lighting Research Center (LRC), in Troy, N.Y., for example, have reported that daylit environments increase occupant productivity and comfort, and provide the mental and visual stimulation necessary to regulate human circadian rhythms (Leslit, R.P., 2003). These findings confirmed the research undertaken across thousands of users in an assortment of building types in different climatic zones around the world shows those users of naturally ventilated and hybrid buildings are more comfortable than or at least as comfortable as, users of an airconditioned buildings (De Dear, Nicols, Roaf et al).

It is most relevant to schools as another study across 434 classrooms from 22 schools showing that carbon dioxide levels (an indicator of ventilation rates) 1,000 parts per million above the outdoor 
concentrations of carbon dioxide was associated with 10 to20 percent increases in student absences. (Shendell, DG, ET.AL. 20014)

Table 2: Responses of Faculty Members, School Official and Students on the Physical Greening of the School

\begin{tabular}{|c|c|c|c|c|c|c|c|c|c|c|}
\hline \multirow{3}{*}{ Place } & \multicolumn{3}{|c|}{ Students } & \multicolumn{3}{|c|}{ Faculty Members } & \multicolumn{3}{|c|}{ School Official } & \multirow[b]{3}{*}{ RANK } \\
\hline & $\mathbf{P}$ & $\mathbf{N}$ & NR & $\mathbf{P}$ & $\mathbf{N}$ & NR & $\mathbf{P}$ & $\mathbf{N}$ & NR & \\
\hline & $\begin{array}{c}\mathbf{f} \\
(\%)\end{array}$ & $\begin{array}{c}\mathbf{f} \\
(\%)\end{array}$ & $\begin{array}{c}\mathbf{f} \\
(\%)\end{array}$ & $\begin{array}{l}F \\
(\%)\end{array}$ & $\begin{array}{c}f \\
(\%)\end{array}$ & $\begin{array}{c}\mathbf{f} \\
(\%)\end{array}$ & $\begin{array}{c}\mathbf{f} \\
(\%)\end{array}$ & $\begin{array}{c}f \\
(\%)\end{array}$ & $\begin{array}{c}f \\
(\%)\end{array}$ & \\
\hline On the entire campus & $\begin{array}{c}35 \\
(42.17)\end{array}$ & $\begin{array}{c}16 \\
(19.28)\end{array}$ & $\begin{array}{c}18 \\
(21.69)\end{array}$ & $\begin{array}{c}9 \\
(10.84)\end{array}$ & 0 & $\begin{array}{c}4 \\
(4.8)\end{array}$ & $\begin{array}{c}1 \\
(1.2)\end{array}$ & 0 & 0 & 1 \\
\hline $\begin{array}{l}\text { In the specific area } \\
\text { inside the campus }\end{array}$ & $\begin{array}{c}33 \\
(39.76)\end{array}$ & $\begin{array}{c}19 \\
(22.89)\end{array}$ & $\begin{array}{c}17 \\
(20.48)\end{array}$ & $\begin{array}{c}5 \\
(6.02)\end{array}$ & $\begin{array}{c}1 \\
(1.2)\end{array}$ & $\begin{array}{c}8 \\
(9.6)\end{array}$ & 0 & 0 & $\begin{array}{c}1 \\
(1.2)\end{array}$ & 2 \\
\hline Indoors only & $\begin{array}{c}25 \\
(30.12)\end{array}$ & $\begin{array}{c}23 \\
(27.7)\end{array}$ & $\begin{array}{c}21 \\
(25.3)\end{array}$ & $\begin{array}{c}4 \\
(4.8)\end{array}$ & $\begin{array}{c}1 \\
(1.2)\end{array}$ & $\begin{array}{c}9 \\
(10.84)\end{array}$ & 0 & 0 & $\begin{array}{c}1 \\
(1.2)\end{array}$ & 4 \\
\hline $\begin{array}{c}\text { In areas outside the } \\
\text { campus }\end{array}$ & $\begin{array}{c}29 \\
(34.9)\end{array}$ & $\begin{array}{c}17 \\
(20.48)\end{array}$ & $\begin{array}{c}23 \\
(27.7)\end{array}$ & $\begin{array}{c}5 \\
(6.02)\end{array}$ & $\begin{array}{c}1 \\
(1.2)\end{array}$ & $\begin{array}{c}8 \\
(9.6)\end{array}$ & 0 & 0 & $\begin{array}{c}1 \\
(1.2)\end{array}$ & 3 \\
\hline TOTAL & $\begin{array}{c}122 \\
(146.98)\end{array}$ & $\begin{array}{c}75 \\
(90.36)\end{array}$ & $\begin{array}{c}79 \\
(75.18)\end{array}$ & $\begin{array}{c}23 \\
(27.7)\end{array}$ & $\begin{array}{c}3 \\
(3.6)\end{array}$ & $\begin{array}{c}29 \\
(34.9)\end{array}$ & $\begin{array}{c}1 \\
(1.2)\end{array}$ & 0 & $\begin{array}{c}3 \\
(3.6)\end{array}$ & \\
\hline
\end{tabular}

Respondents agreed that physical greening is undertaken in the school. Most respondents agreed that physical greening is practiced in the entire campus, in specific areas inside the campus and also in the areas outside the campus.

\subsection{Lights and Electricity}

Table 3: Response of Faculty Members, School official, and Students on the Source of Lighting in the School

\begin{tabular}{|c|c|c|c|c|c|c|c|c|c|c|}
\hline \multirow{3}{*}{ Source of Light } & \multicolumn{3}{|c|}{ Students } & \multicolumn{3}{|c|}{ Faculty Members } & \multicolumn{3}{|c|}{ School Official } & \multirow[b]{3}{*}{ RANK } \\
\hline & $\mathbf{P}$ & $\mathbf{N}$ & NR & $\mathbf{P}$ & $\mathbf{N}$ & NR & $\mathbf{P}$ & $\mathbf{N}$ & NR & \\
\hline & $\begin{array}{c}\mathbf{f} \\
(\%)\end{array}$ & $\begin{array}{c}\mathbf{f} \\
(\%)\end{array}$ & $\begin{array}{c}\mathbf{f} \\
(\%)\end{array}$ & $\begin{array}{c}\mathbf{f} \\
(\%)\end{array}$ & $\begin{array}{c}\mathbf{f} \\
(\%)\end{array}$ & $\begin{array}{c}f \\
(\%)\end{array}$ & $\begin{array}{c}\mathbf{f} \\
(\%)\end{array}$ & $\begin{array}{c}f \\
(\%)\end{array}$ & $\begin{array}{c}f \\
(\%)\end{array}$ & \\
\hline Fluorescent & $\begin{array}{c}34 \\
(40.96)\end{array}$ & $\begin{array}{c}17 \\
(20.48)\end{array}$ & $\begin{array}{c}18 \\
(21.69)\end{array}$ & $\begin{array}{c}5 \\
(6.02) \\
\end{array}$ & $\begin{array}{c}1 \\
(1.2)\end{array}$ & $\begin{array}{c}8 \\
(9.6)\end{array}$ & $\begin{array}{c}1 \\
(1.2)\end{array}$ & 0 & 0 & 1 \\
\hline Incandescent & $\begin{array}{c}13 \\
(15.66)\end{array}$ & $\begin{array}{c}25 \\
(30.12)\end{array}$ & $\begin{array}{c}31 \\
(37.35)\end{array}$ & $\begin{array}{c}2 \\
(2.4)\end{array}$ & $\begin{array}{c}2 \\
(2.4)\end{array}$ & $\begin{array}{c}10 \\
(12.05)\end{array}$ & 0 & 0 & $\begin{array}{c}1 \\
(1.2)\end{array}$ & 2 \\
\hline TOTAL & $\begin{array}{c}47 \\
(56.63)\end{array}$ & $\begin{array}{c}42 \\
(50.6)\end{array}$ & $\begin{array}{c}49 \\
(59.04)\end{array}$ & $\begin{array}{c}7 \\
(8.4)\end{array}$ & $\begin{array}{c}3 \\
(3.6)\end{array}$ & $\begin{array}{c}18 \\
(21.69)\end{array}$ & $\begin{array}{c}1 \\
(1.2)\end{array}$ & $\begin{array}{c}1 \\
(1.2)\end{array}$ & $\begin{array}{c}1 \\
(1.2)\end{array}$ & \\
\hline
\end{tabular}

A majority of the respondents agreed that the fluorescent lamps were the most commonly used source of the light wherein 34 or $40.96 \%$ coming from the students, $6.02 \%$ from the faculty members and also the school officials gave a positive response. This means that the incandescent bulbs were still used in the other parts of the school. It further indicates that more energy is conserved if fluorescent lamps are used because it has lesser voltage requirement as compared to incandescent lamps. This further means that the school is not yet aware on the importance of LED lights compared to flourescent lights in terms of energy conservation measures. 


\subsection{Water}

Table 4: Responses of Faculty Members, School Official, and Students on the Source of Water for Everyday Use

\begin{tabular}{|c|c|c|c|c|c|c|c|c|c|c|}
\hline \multirow{3}{*}{ Source of water } & \multicolumn{3}{|c|}{ Students } & \multicolumn{3}{|c|}{ Faculty Members } & \multicolumn{3}{|c|}{ School Official } & \multirow[b]{3}{*}{ RANK } \\
\hline & $\mathbf{P}$ & $\mathbf{N}$ & NR & $\mathbf{P}$ & $\mathbf{N}$ & NR & $\mathbf{P}$ & $\mathbf{N}$ & NR & \\
\hline & $\begin{array}{c}\mathbf{f} \\
(\%)\end{array}$ & $\begin{array}{c}\mathbf{f} \\
(\%)\end{array}$ & $\begin{array}{c}\mathbf{f} \\
(\%)\end{array}$ & $\begin{array}{l}\mathbf{F} \\
(\%)\end{array}$ & $\begin{array}{c}\mathbf{f} \\
(\%)\end{array}$ & $\begin{array}{c}\mathbf{f} \\
(\%)\end{array}$ & $\begin{array}{c}\mathbf{f} \\
(\%)\end{array}$ & $\begin{array}{c}\mathbf{f} \\
(\%)\end{array}$ & $\begin{array}{c}\mathbf{f} \\
(\%)\end{array}$ & \\
\hline $\begin{array}{c}\text { From local water work } \\
\text { through pipe }\end{array}$ & $\begin{array}{c}39 \\
(46.99)\end{array}$ & $\begin{array}{c}17 \\
(20.48)\end{array}$ & $\begin{array}{c}11 \\
(13.25)\end{array}$ & $\begin{array}{c}10 \\
(12.05)\end{array}$ & 0 & $\begin{array}{c}4 \\
(4.8) \\
\end{array}$ & $\begin{array}{c}1 \\
(1.2) \\
\end{array}$ & 0 & 0 & 1 \\
\hline $\begin{array}{c}\text { Local water works but } \\
\text { stored in tank }\end{array}$ & $\begin{array}{c}24 \\
(28.9)\end{array}$ & $\begin{array}{c}26 \\
(31.33) \\
\end{array}$ & $\begin{array}{c}19 \\
(22.87)\end{array}$ & $\begin{array}{c}1 \\
(1.2)\end{array}$ & $\begin{array}{c}2 \\
(2.4)\end{array}$ & $\begin{array}{c}11 \\
(13.25)\end{array}$ & 0 & 0 & $\begin{array}{c}1 \\
(1.2) \\
\end{array}$ & 2 \\
\hline From artesian well & $\begin{array}{c}19 \\
(22.87)\end{array}$ & $\begin{array}{c}27 \\
(32.5)\end{array}$ & $\begin{array}{c}23 \\
(27.7)\end{array}$ & $\begin{array}{c}4 \\
(4.8) \\
\end{array}$ & $\begin{array}{c}2 \\
(2.4)\end{array}$ & $\begin{array}{c}8 \\
(9.67)\end{array}$ & 0 & 0 & $\begin{array}{c}1 \\
(1.2)\end{array}$ & 3 \\
\hline TOTAL & $\begin{array}{c}82 \\
(98.8) \\
\end{array}$ & $\begin{array}{c}70 \\
(84.3) \\
\end{array}$ & $\begin{array}{c}53 \\
(63.9) \\
\end{array}$ & $\begin{array}{c}15 \\
(18.07) \\
\end{array}$ & $\begin{array}{c}4 \\
(4.8) \\
\end{array}$ & $\begin{array}{c}23 \\
(27.7) \\
\end{array}$ & $\begin{array}{c}1 \\
(1.2) \\
\end{array}$ & 0 & $\begin{array}{c}2 \\
(2.4) \\
\end{array}$ & \\
\hline
\end{tabular}

The common source of water was from the local water works with $46.99 \%$ from the students, $12.05 \%$ from the faculty members and the school officials gave a positive response. Ranked second was that the water was sourced from the local water works but stored in the tank with 24 or $28.9 \%$ from the students, 1 or $1.2 \%$ came from the faculty members and no response from the school officials. Lowest in the rank was the artesian well, 19 or $22.89 \%$ from the students, 4 or $4.8 \%$ from the faculty members while the school official had no response.

\subsection{Office Machines and Equipment}

Table 5: Responses of Faculty Members, School Official, and Students on Office Machine and Equipment

\begin{tabular}{|c|c|c|c|c|c|c|c|c|c|c|}
\hline \multirow{3}{*}{$\begin{array}{l}\text { Machines/ } \\
\text { Equipment }\end{array}$} & \multicolumn{3}{|c|}{ Students } & \multicolumn{3}{|c|}{ Faculty Members } & \multicolumn{3}{|c|}{ School Official } & \multirow[b]{3}{*}{ RANK } \\
\hline & $\mathbf{P}$ & $\mathbf{N}$ & NR & $\mathbf{P}$ & $\mathbf{N}$ & NR & $\mathbf{P}$ & $\mathbf{N}$ & NR & \\
\hline & $\begin{array}{c}f \\
(\%)\end{array}$ & $\begin{array}{c}f \\
(\%)\end{array}$ & $\begin{array}{c}\mathbf{f} \\
(\%)\end{array}$ & $\begin{array}{l}\mathbf{F} \\
(\%)\end{array}$ & $\begin{array}{c} \\
(\%)\end{array}$ & $\begin{array}{c} \\
(\%)\end{array}$ & $\begin{array}{c}f \\
(\%)\end{array}$ & $\begin{array}{c}f \\
(\%)\end{array}$ & $\begin{array}{c}\mathbf{f} \\
(\%)\end{array}$ & \\
\hline $\begin{array}{c}\text { Use of air conditioner } \\
\text { regularly }\end{array}$ & 0 & 0 & 0 & 0 & 0 & 0 & 0 & 0 & 0 & 3 \\
\hline $\begin{array}{c}\text { Using of fans } \\
\text { whenever possible }\end{array}$ & $\begin{array}{c}26 \\
(31.33)\end{array}$ & $\begin{array}{c}23 \\
(27.7)\end{array}$ & $\begin{array}{c}20 \\
(24.9)\end{array}$ & $\begin{array}{c}3 \\
(3.6)\end{array}$ & $\begin{array}{c}1 \\
(1.2)\end{array}$ & $\begin{array}{c}10 \\
(12.05)\end{array}$ & $\begin{array}{c}1 \\
(1.2)\end{array}$ & 0 & 0 & 2 \\
\hline $\begin{array}{l}\text { Office machine turned } \\
\text { off when not in used }\end{array}$ & $\begin{array}{c}39 \\
(46.99)\end{array}$ & $\begin{array}{c}16 \\
(19.3)\end{array}$ & $\begin{array}{c}14 \\
(16.87)\end{array}$ & $\begin{array}{c}6 \\
(7.23)\end{array}$ & $\begin{array}{c}2 \\
(2.4)\end{array}$ & $\begin{array}{c}6 \\
(7.23)\end{array}$ & $\begin{array}{c}1 \\
(1.2)\end{array}$ & 0 & 0 & 1 \\
\hline TOTAL & $\begin{array}{c}65 \\
(78.3)\end{array}$ & $\begin{array}{c}39 \\
(46.99)\end{array}$ & $\begin{array}{c}34 \\
(40.96)\end{array}$ & $\begin{array}{c}9 \\
(10.84)\end{array}$ & $\begin{array}{c}3 \\
(3.6)\end{array}$ & $\begin{array}{c}16 \\
(19.3)\end{array}$ & $\begin{array}{c}2 \\
(2.4)\end{array}$ & 0 & 0 & \\
\hline
\end{tabular}

The school had its way of conserving energy, with its machine being turned off when not in use wherein 39 or $46.99 \%$ from the students, 6 or $7.23 \%$ from the faculty members and the school official gave a positive response. It is followed by the use of fans wherever possible and only 26 or $31.33 \%$ from the students, 3 or $3.6 \%$ from the faculty member and the school official gave a positive response. This means that energy measures were practiced in their offices/school premises even if it is not part of their environmental policy. 


\subsection{Paper}

Table 6: Responses of Faculty Members, School Official, and Students on the kind of Paper Preferred in the School

\begin{tabular}{|c|c|c|c|c|c|c|c|}
\hline \multirow{2}{*}{$\begin{array}{c}\text { Kind of } \\
\text { Paper }\end{array}$} & \multicolumn{3}{|c|}{ Faculty Members } & \multicolumn{2}{c|}{ School Official } & \multirow{2}{*}{} \\
\cline { 2 - 7 } & $\mathbf{P}$ & $\mathbf{N}$ & $\mathbf{N R}$ & $\mathbf{P}$ & $\mathbf{N}$ & $\mathbf{N R}$ & \multirow{2}{*}{ RANK } \\
\cline { 2 - 7 } & $\mathbf{F}$ & $\mathbf{f}$ & $\mathbf{f}$ & $\mathbf{f}$ & $\mathbf{f}$ & $\mathbf{f}$ \\
$(\boldsymbol{\%})$ & $(\boldsymbol{\%})$ & $(\boldsymbol{\%})$ & $(\boldsymbol{\%})$ & $(\%)$ & $(\%)$ & \\
\hline White & $\begin{array}{c}14 \\
(16.87)\end{array}$ & 0 & 0 & $(1.2)$ & 0 & 0 & 1 \\
\hline Colored & 6 & 3 & 5 & & & 1 & \\
& $(7.23)$ & $(3.6)$ & $(6.02)$ & 0 & 0 & $(1.2)$ & 2 \\
\hline New print quality & 9 & 1 & 4 & 1 & & & \\
& $(10.84)$ & $(1.2)$ & $(4.8)$ & $(1.2)$ & 0 & 0 & 3 \\
\hline $\mathbf{2 9}$ & 4 & 9 & 2 & & 1 & & \\
$(\mathbf{3 4 . 9})$ & $(4.8)$ & $(10.84)$ & $(2.4)$ & 0 & $(1.2)$ & \\
\hline
\end{tabular}

On the kind of paper preferred in the school, white paper ranked highest, 62 or $74.7 \%$ from the students, 14 or $16.87 \%$ from the faculty members and the school official gave a positive response. Ranked second was colored paper, 41 or $49.4 \%, 35$ or $42.17 \%$ from the students, 6 or $7.23 \%$ from the faculty members and there was no response from the school official. The last rank was new print quality with 38 or $45.78 \%, 28$ or $33.7 \%$ from the students, 9 or $10.84 \%$ from the faculty members and the school official gave a positive response. This only means that students, faculty and official still use white paper in their daily transactions.

Table 7: Responses of Faculty Members, School Official, and Students on Paper Recycling, Reuse and Reduction Program in the School

\begin{tabular}{|c|c|c|c|c|c|c|c|c|c|c|}
\hline \multirow{3}{*}{$\begin{array}{l}\text { Type of paper } \\
\text { recycling }\end{array}$} & \multicolumn{3}{|c|}{ Students } & \multicolumn{3}{|c|}{ Faculty Members } & \multicolumn{3}{|c|}{ School Official } & \multirow[b]{3}{*}{ RANK } \\
\hline & $\mathbf{P}$ & $\mathbf{N}$ & NR & $\mathbf{P}$ & $\mathbf{N}$ & NR & $\mathbf{P}$ & $\mathbf{N}$ & NR & \\
\hline & $\begin{array}{c}\mathbf{f} \\
(\%)\end{array}$ & $\begin{array}{c}f \\
(\%)\end{array}$ & $\begin{array}{c} \\
(\%)\end{array}$ & $\begin{array}{l}\mathbf{F} \\
(\%)\end{array}$ & $\begin{array}{c}\mathbf{f} \\
(\%)\end{array}$ & $\begin{array}{c}\mathbf{f} \\
(\%)\end{array}$ & $\begin{array}{c}f \\
(\%)\end{array}$ & $\begin{array}{c}\mathbf{f} \\
(\%)\end{array}$ & $\begin{array}{c}\mathbf{f} \\
(\%)\end{array}$ & \\
\hline $\begin{array}{c}\text { Use of paper in } \\
\text { photocopying } \\
\text { articles }\end{array}$ & $\begin{array}{c}42 \\
(50.6)\end{array}$ & $\begin{array}{c}16 \\
(19.3)\end{array}$ & $\begin{array}{c}11 \\
(13.25)\end{array}$ & $\begin{array}{c}12 \\
(14.6)\end{array}$ & 0 & $\begin{array}{c}2 \\
(2.4)\end{array}$ & 0 & 0 & $\begin{array}{c}1 \\
(1.2)\end{array}$ & 1 \\
\hline $\begin{array}{c}\text { Internal } \\
\text { communication } \\
\end{array}$ & $\begin{array}{c}20 \\
(24.19) \\
\end{array}$ & $\begin{array}{c}28 \\
(33.73) \\
\end{array}$ & $\begin{array}{c}21 \\
(25.3) \\
\end{array}$ & $\begin{array}{c}9 \\
(10.84) \\
\end{array}$ & 0 & $\begin{array}{c}5 \\
(6.02) \\
\end{array}$ & $\begin{array}{c}1 \\
(1.2) \\
\end{array}$ & 0 & 0 & 3 \\
\hline $\begin{array}{l}\text { Recycling use paper } \\
\text { into new product }\end{array}$ & $\begin{array}{c}32 \\
(38.55) \\
\end{array}$ & $\begin{array}{c}19 \\
(22.89) \\
\end{array}$ & $\begin{array}{c}18 \\
(21.69) \\
\end{array}$ & $\begin{array}{c}7 \\
(8.43) \\
\end{array}$ & $\begin{array}{c}1 \\
(1.2)\end{array}$ & $\begin{array}{c}6 \\
(7.23) \\
\end{array}$ & $\begin{array}{c}1 \\
(1.2)\end{array}$ & 0 & 0 & 2 \\
\hline TOTAL & $\begin{array}{c}94 \\
(113.25) \\
\end{array}$ & $\begin{array}{c}63 \\
(75.9) \\
\end{array}$ & $\begin{array}{c}50 \\
(60.24) \\
\end{array}$ & $\begin{array}{c}28 \\
(33.73) \\
\end{array}$ & $\begin{array}{c}1 \\
(1.2) \\
\end{array}$ & $\begin{array}{c}13 \\
(15.66) \\
\end{array}$ & $\begin{array}{c}2 \\
(2.4) \\
\end{array}$ & 0 & $\begin{array}{c}1 \\
(1.2) \\
\end{array}$ & \\
\hline
\end{tabular}

On paper recycling, reuse or reduction program being observed in the school, use of paper in photocopying articles was at 54 or $65.1 \%$ of the respondents, 42 or $50.6 \%$ from the students, 12 or $14.46 \%$ from the faculty members and the school official had no response. Recycling used paper into new product with 32 or $38.55 \%$ from the students, 7 or $8.43 \%$ from the faculty members, and the school official gave a positive response. Reuse or papers for internal communication was ranked last 20 or $20.9 \%$, from the students, 9 or $10.54 \%$ from the faculty members and the school official gave a positive response. Paper recycling is a practice implemented in the school even if it is not part of their policy. 


\subsection{The Canteen}

Table 8: Responses of Faculty Members, School Official and Students on Disposing Wet Waste

\begin{tabular}{|c|c|c|c|c|c|c|c|c|c|c|}
\hline \multirow{3}{*}{$\begin{array}{c}\text { Way in segregating } \\
\text { waste }\end{array}$} & \multicolumn{3}{|c|}{ Students } & \multicolumn{3}{|c|}{ Faculty Members } & \multicolumn{3}{|c|}{ School Official } & \multirow[b]{3}{*}{ RANK } \\
\hline & $\mathbf{P}$ & $\mathbf{N}$ & NR & $\mathbf{P}$ & $\mathbf{N}$ & NR & $\mathbf{P}$ & $\mathbf{N}$ & NR & \\
\hline & $\begin{array}{l}\mathbf{f} \\
(\%)\end{array}$ & $\begin{array}{l}\mathbf{f} \\
(\%)\end{array}$ & $\begin{array}{c}\mathbf{f} \\
(\%)\end{array}$ & $\begin{array}{l}\mathbf{F} \\
(\%)\end{array}$ & $\begin{array}{c}\text { f } \\
(\%)\end{array}$ & $\begin{array}{c}\mathbf{f} \\
(\%)\end{array}$ & $\begin{array}{c}f \\
(\%)\end{array}$ & $\begin{array}{c}\mathbf{f} \\
(\%)\end{array}$ & $\begin{array}{c}f \\
(\%)\end{array}$ & \\
\hline $\begin{array}{c}\text { Segregated and } \\
\text { prepared for } \\
\text { collection }\end{array}$ & $\begin{array}{c}29 \\
(34.9)\end{array}$ & $\begin{array}{c}24 \\
(28.9)\end{array}$ & $\begin{array}{c}16 \\
(19.3)\end{array}$ & $\begin{array}{c}4 \\
(4.8)\end{array}$ & $\begin{array}{c}1 \\
(1.2)\end{array}$ & $\begin{array}{c}9 \\
(10.84)\end{array}$ & $\begin{array}{c}1 \\
(1.2)\end{array}$ & 0 & 0 & 2 \\
\hline $\begin{array}{l}\text { Biodegradable waste } \\
\text { composted right in } \\
\text { the school compound }\end{array}$ & $\begin{array}{c}32 \\
(38.55)\end{array}$ & $\begin{array}{c}16 \\
(19.3)\end{array}$ & $\begin{array}{c}21 \\
(25.3)\end{array}$ & $\begin{array}{c}6 \\
(7.23)\end{array}$ & 0 & $\begin{array}{c}8 \\
(9.6)\end{array}$ & $\begin{array}{c}1 \\
(1.2)\end{array}$ & 0 & 0 & 1 \\
\hline Sent to recyclers & $\begin{array}{c}25 \\
(30.12) \\
\end{array}$ & $\begin{array}{c}21 \\
(25.3)\end{array}$ & $\begin{array}{c}23 \\
(27.7) \\
\end{array}$ & $\begin{array}{c}1 \\
(1.2) \\
\end{array}$ & $\begin{array}{c}1 \\
(1.2) \\
\end{array}$ & $\begin{array}{c}12 \\
(14.46) \\
\end{array}$ & 0 & 0 & $\begin{array}{c}1 \\
(1.20 \\
\end{array}$ & 3 \\
\hline TOTAL & $\begin{array}{c}86 \\
(103.6)\end{array}$ & $\begin{array}{c}61 \\
(73.49)\end{array}$ & $\begin{array}{c}60 \\
(72.29)\end{array}$ & $\begin{array}{c}11 \\
(13.25)\end{array}$ & $\begin{array}{c}2 \\
(2.4)\end{array}$ & $\begin{array}{c}29 \\
(34.9)\end{array}$ & $\begin{array}{c}2 \\
(2.4)\end{array}$ & 0 & $\begin{array}{c}1 \\
(1.2)\end{array}$ & \\
\hline
\end{tabular}

Biodegradable waste was composted right in the school compound, wherein 39 or $46.99 \%$, 32 or $38.55 \%$ from the students, 6 or $7.23 \%$ from the faculty members and the school official gave a positive response. Segregated and prepared for collection with 29 or $34.93 \%$ from the students, 4 or $4.8 \%$ from the faculty members and the school official gave a positive response set to recyclers with 25 or $30.12 \%$ from the students, only 1 or $1.2 \%$ from the school official while school official had no response. Waste segregation and composting was being practiced by the respondents especially that the school campus favored wide area for composting of biodegrable wastes.

Table 9: Responses of Faculty Members, School Official, and Students on Materials not allowed or Discouraged in the School Canteen

\begin{tabular}{|c|c|c|c|c|c|c|c|c|c|c|}
\hline \multirow{3}{*}{ Materials } & \multicolumn{3}{|c|}{ Students } & \multicolumn{3}{|c|}{ Faculty Members } & \multicolumn{3}{|c|}{ School Official } & \multirow[b]{3}{*}{ RANK } \\
\hline & $\mathbf{P}$ & $\mathbf{N}$ & NR & $\mathbf{P}$ & $\mathbf{N}$ & NR & $\mathbf{P}$ & $\mathbf{N}$ & NR & \\
\hline & $\begin{array}{c}\mathbf{f} \\
(\%)\end{array}$ & $\begin{array}{c}\mathbf{f} \\
(\%)\end{array}$ & $\begin{array}{c}f \\
(\%)\end{array}$ & $\begin{array}{c}\mathrm{F} \\
(\%)\end{array}$ & $\begin{array}{c} \\
(\%)\end{array}$ & $\begin{array}{c}\mathbf{f} \\
(\%)\end{array}$ & $\begin{array}{c} \\
(\%)\end{array}$ & $\begin{array}{c} \\
(\%)\end{array}$ & $\begin{array}{c}f \\
(\%)\end{array}$ & \\
\hline Disposable cups & $\begin{array}{c}27 \\
(32.53)\end{array}$ & $\begin{array}{c}22 \\
(26.5)\end{array}$ & $\begin{array}{c}20 \\
(24.09)\end{array}$ & $\begin{array}{c}5 \\
(6.02)\end{array}$ & $\begin{array}{c}1 \\
(1.2)\end{array}$ & $\begin{array}{c}8 \\
(9.6)\end{array}$ & $\begin{array}{c}1 \\
(1.2)\end{array}$ & 0 & 0 & 2 \\
\hline Disposable plates & $\begin{array}{c}26 \\
(31.33)\end{array}$ & $\begin{array}{c}22 \\
(26.5)\end{array}$ & $\begin{array}{c}21 \\
(25.3)\end{array}$ & $\begin{array}{c}5 \\
(6.02)\end{array}$ & $\begin{array}{c}1 \\
(1.2)\end{array}$ & $\begin{array}{c}8 \\
(9.6)\end{array}$ & $\begin{array}{c}1 \\
(1.2)\end{array}$ & 0 & 0 & 3 \\
\hline $\begin{array}{c}\text { Disposable Plastic } \\
\text { Utensils }\end{array}$ & $\begin{array}{c}30 \\
(36.14)\end{array}$ & $\begin{array}{c}19 \\
(22.89)\end{array}$ & $\begin{array}{c}20 \\
(24.09)\end{array}$ & $\begin{array}{c}4 \\
(4.8)\end{array}$ & $\begin{array}{c}1 \\
(1.2)\end{array}$ & $\begin{array}{c}9 \\
(10.84)\end{array}$ & $\begin{array}{c}1 \\
(1.2)\end{array}$ & 0 & 0 & 1 \\
\hline Paper Napkins & $\begin{array}{c}13 \\
(15.66)\end{array}$ & $\begin{array}{c}33 \\
(39.76)\end{array}$ & $\begin{array}{c}23 \\
(27.7)\end{array}$ & $\begin{array}{c}1 \\
(1.2)\end{array}$ & $\begin{array}{c}2 \\
(2.4)\end{array}$ & $\begin{array}{c}11 \\
(13.25)\end{array}$ & 0 & 0 & $\begin{array}{c}1 \\
(1.2)\end{array}$ & 4 \\
\hline Plastic Wrapper & $\begin{array}{c}27 \\
(32.53)\end{array}$ & $\begin{array}{c}21 \\
(25.3)\end{array}$ & $\begin{array}{c}21 \\
(25.3)\end{array}$ & $\begin{array}{c}4 \\
(4.8)\end{array}$ & $\begin{array}{c}2 \\
(2.4)\end{array}$ & $\begin{array}{c}8 \\
(9.6)\end{array}$ & $\begin{array}{c}1 \\
(1.2)\end{array}$ & 0 & 0 & 2 \\
\hline TOTAL & $\begin{array}{c}123 \\
(148.12)\end{array}$ & $\begin{array}{c}117 \\
(140.96)\end{array}$ & $\begin{array}{c}105 \\
(126.5) \\
\end{array}$ & $\begin{array}{c}19 \\
(22.89) \\
\end{array}$ & $\begin{array}{c}7 \\
(8.43)\end{array}$ & $\begin{array}{c}44 \\
(53)\end{array}$ & $\begin{array}{c}4 \\
(4.8)\end{array}$ & 0 & $\begin{array}{c}1 \\
(1.2)\end{array}$ & \\
\hline
\end{tabular}

As to the materials that were discouraged in the canteen, disposable plastic utensils ranked highest wherein 30 or $36.14 \%$ came from the students, 4 or $4.8 \%$ from the faculty members, and the school official gave a positive response. This is followed by the plastic wrappers and disposable cups with 27 or $32.53 \%$ coming from the students, 4 or $4.8 \%$ from faculty members, and the school official gave a positive response; disposable plates ranked third with 26 or $31.33 \%$ from the student, 5 or 
$6.02 \%$ coming from the faculty members, the school official give a positive response; and finally, paper napkins with 13 or $15.66 \%$ coming from the students, only 1 or $1.2 \%$ from the faculty members, and no response from the school official.

\subsection{General Waste Management}

Table 10: Responses of Faculty Members, School Official, and Students on the Practice of Waste Segregation in the School

\begin{tabular}{|c|c|c|c|c|}
\hline \multirow{2}{*}{ Responses } & Students & Faculty Members & School Official & \multirow{2}{*}{ RANK } \\
\cline { 2 - 4 } Positive & $\mathbf{f}(\%)$ & $\mathbf{f}(\%)$ & $\mathbf{f}(\%)$ & 1 \\
\hline Negative & $\begin{array}{c}51 \\
(61.45)\end{array}$ & $\begin{array}{c}8 \\
(9.6)\end{array}$ & $(1.2)$ & 1 \\
\hline $\begin{array}{c}6 \\
\text { No }\end{array}$ & $12.23)$ & 0 & 0 & 2 \\
\hline Response & $(14.45)$ & 6 & 0 & 3 \\
\hline TOTAL & 69 & $(7.23)$ & 1 & \\
$(83.13)$ & $(16.86)$ & $(1.2)$ & \\
\hline
\end{tabular}

Waste segregation is practiced in the school campus, with 51 favored respondents or $61.45 \%$ coming from the students, 8 or $9.6 \%$ from the faculty members and the school official gave a positive response. Only 60 respondents agreed that waste segregation is practiced in the school or about $72.29 \%$ of the total positive response.

Table 11: Responses of Faculty Members, School Official, and Students on Separating Containers from Biodegradable and Non-biodegradable Waste

\begin{tabular}{|c|c|c|c|c|c|c|c|c|c|c|}
\hline \multirow{3}{*}{ Provided in } & \multicolumn{3}{|c|}{ Students } & \multicolumn{3}{|c|}{ Faculty Members } & \multicolumn{3}{|c|}{ School Official } & \multirow[b]{3}{*}{ RANK } \\
\hline & $\mathbf{P}$ & $\mathbf{N}$ & NR & $\mathbf{P}$ & $\mathbf{N}$ & NR & $\mathbf{P}$ & $\mathbf{N}$ & NR & \\
\hline & $\begin{array}{c}\mathbf{f} \\
(\%)\end{array}$ & $\begin{array}{c}f \\
(\%)\end{array}$ & $\begin{array}{l}\mathbf{f} \\
(\%)\end{array}$ & $\begin{array}{c}\mathbf{F} \\
(\%)\end{array}$ & $\begin{array}{c}f \\
(\%)\end{array}$ & $\begin{array}{c}f \\
(\%)\end{array}$ & $\begin{array}{c}\mathbf{f} \\
(\%)\end{array}$ & $\begin{array}{c}\mathbf{f} \\
(\%)\end{array}$ & $\begin{array}{c}f \\
(\%)\end{array}$ & \\
\hline The entire campus & $\begin{array}{c}50 \\
(60.24) \\
\end{array}$ & $\begin{array}{c}11 \\
(13.25)\end{array}$ & $\begin{array}{c}8 \\
(9.6)\end{array}$ & $\begin{array}{c}11 \\
(13.25)\end{array}$ & 0 & $\begin{array}{c}3 \\
(3.6) \\
\end{array}$ & $\begin{array}{c}1 \\
(1.2)\end{array}$ & 0 & 0 & 1 \\
\hline Selected Areas & $\begin{array}{c}29 \\
(34.93)\end{array}$ & $\begin{array}{c}11 \\
(13.25)\end{array}$ & $\begin{array}{c}29 \\
(34.93)\end{array}$ & $\begin{array}{c}2 \\
(2.4)\end{array}$ & $\begin{array}{c}1 \\
(1.2)\end{array}$ & $\begin{array}{c}11 \\
(13.25)\end{array}$ & 0 & 0 & $\begin{array}{c}1 \\
(1.2)\end{array}$ & 2 \\
\hline TOTAL & $\begin{array}{c}79 \\
(95.18)\end{array}$ & $\begin{array}{c}22 \\
(26.5)\end{array}$ & $\begin{array}{c}37 \\
(44.58)\end{array}$ & $\begin{array}{c}13 \\
(15.66)\end{array}$ & $\begin{array}{c}1 \\
(1.2)\end{array}$ & $\begin{array}{c}14 \\
(16.87)\end{array}$ & $\begin{array}{c}1 \\
(1.2)\end{array}$ & 0 & $\begin{array}{c}1 \\
(1.20 \\
\end{array}$ & \\
\hline
\end{tabular}

Containers for biodegradable and non-biodegradable waste were provided on in selected areas with $34.93 \%$ from the students, $34.93 \%$ from the faculty members, and no response from the school official. A total of 62 respondents or $74.7 \%$ agreed that containers were provided in the entire campus. It further reveals that containers for waste were not provided in the entire campus but only in selected areas. 
Table 12: Responses of Faculty Members, School Official, and Students on Waste Segregation for Biodegradable Materials

\begin{tabular}{|c|c|c|c|c|c|c|c|c|c|c|}
\hline \multirow[b]{3}{*}{ Practices } & \multicolumn{3}{|c|}{ Students } & \multicolumn{3}{|c|}{ Faculty Members } & \multicolumn{3}{|c|}{ School Official } & \multirow[b]{3}{*}{ RANK } \\
\hline & $\mathbf{P}$ & $\mathbf{N}$ & NR & $\mathbf{P}$ & $\mathbf{N}$ & NR & $\mathbf{P}$ & $\mathbf{N}$ & NR & \\
\hline & $\begin{array}{c}f \\
(\%)\end{array}$ & $\begin{array}{c}f \\
(\%)\end{array}$ & $\begin{array}{c}\mathbf{f} \\
(\%)\end{array}$ & $\begin{array}{l}F \\
(\%)\end{array}$ & $\begin{array}{c}\text { f } \\
(\%)\end{array}$ & $\begin{array}{c}\mathbf{f} \\
(\%)\end{array}$ & $\begin{array}{c}\mathbf{f} \\
(\%)\end{array}$ & $\begin{array}{c} \\
(\%)\end{array}$ & $\begin{array}{c}f \\
(\%)\end{array}$ & \\
\hline Made to Compose & $\begin{array}{c}40 \\
(48.19)\end{array}$ & $\begin{array}{c}17 \\
(20.48)\end{array}$ & $\begin{array}{c}12 \\
(14.46)\end{array}$ & $\begin{array}{c}8 \\
(9.6)\end{array}$ & 0 & $\begin{array}{c}6 \\
(7.23)\end{array}$ & $\begin{array}{c}1 \\
(1.2)\end{array}$ & 0 & 0 & 1 \\
\hline Send to Hog Raisers & $\begin{array}{c}16 \\
(19.27)\end{array}$ & $\begin{array}{c}32 \\
(38.55)\end{array}$ & $\begin{array}{c}21 \\
(25.30)\end{array}$ & $\begin{array}{c}1 \\
(1.2)\end{array}$ & $\begin{array}{c}2 \\
(2.4)\end{array}$ & $\begin{array}{c}11 \\
(13.25)\end{array}$ & 0 & 0 & $\begin{array}{c}1 \\
(1.2)\end{array}$ & 3 \\
\hline Thrown away & $\begin{array}{c}27 \\
(32.5) \\
\end{array}$ & $\begin{array}{c}23 \\
(27.7) \\
\end{array}$ & $\begin{array}{c}19 \\
(19.27) \\
\end{array}$ & $\begin{array}{c}6 \\
(7.23) \\
\end{array}$ & 0 & $\begin{array}{c}8 \\
(9.6) \\
\end{array}$ & 0 & 0 & $\begin{array}{c}1 \\
(1.2) \\
\end{array}$ & 2 \\
\hline TOTAL & $\begin{array}{c}87 \\
(100) \\
\end{array}$ & $\begin{array}{c}72 \\
(86.7) \\
\end{array}$ & $\begin{array}{c}52 \\
(62.65) \\
\end{array}$ & $\begin{array}{c}15 \\
(18.1)\end{array}$ & $\begin{array}{c}2 \\
(2.4) \\
\end{array}$ & $\begin{array}{c}25 \\
(30.1)\end{array}$ & $\begin{array}{c}1 \\
(1.2)\end{array}$ & 0 & $\begin{array}{c}2 \\
(2.4) \\
\end{array}$ & \\
\hline
\end{tabular}

On the disposal of biodegradable material, 27 or $32.5 \%$ came from the students, 6 favored that biodegradable wastes were thrown away; while 49 or $59.04 \%$ favored that biodegradable wastes were composted; and 17 or $20.48 \%$ agreed that biodegradable (food) wastes were sent to hog raisers, wherein 16 or $19.27 \%$ came from the students, 1 or $1.2 \%$ from the faculty members, and no response from the school official.

Table 13: Responses of Faculty Members, School Official, and Students on Waste Segregation for Non Biodegradable Materials

\begin{tabular}{|c|c|c|c|c|c|c|c|c|c|c|}
\hline \multirow[b]{3}{*}{ Ways/Practices } & \multicolumn{3}{|c|}{ Students } & \multicolumn{3}{|c|}{ Faculty Members } & \multicolumn{3}{|c|}{ School Official } & \multirow[b]{3}{*}{ RANK } \\
\hline & $\mathbf{P}$ & $\mathbf{N}$ & NR & $\mathbf{P}$ & $\mathbf{N}$ & NR & $\mathbf{P}$ & $\mathbf{N}$ & NR & \\
\hline & $\begin{array}{c}\mathbf{f} \\
(\%)\end{array}$ & $\begin{array}{c}\mathbf{f} \\
(\%)\end{array}$ & $\begin{array}{c}\mathbf{f} \\
(\%)\end{array}$ & $\begin{array}{c}\mathbf{F} \\
(\%)\end{array}$ & $\begin{array}{c}\mathbf{f} \\
(\%)\end{array}$ & $\begin{array}{c}\mathbf{f} \\
(\%)\end{array}$ & $\begin{array}{c}\mathbf{f} \\
(\%)\end{array}$ & $\begin{array}{c}\mathbf{f} \\
(\%)\end{array}$ & $\begin{array}{c}\mathbf{f} \\
(\%)\end{array}$ & \\
\hline Sent to Recyclers & $\begin{array}{c}37 \\
(44.58)\end{array}$ & $\begin{array}{c}25 \\
(30.12)\end{array}$ & $\begin{array}{c}7 \\
(8.43)\end{array}$ & $\begin{array}{c}2 \\
(2.4)\end{array}$ & $\begin{array}{c}1 \\
(1.2)\end{array}$ & $\begin{array}{c}11 \\
(13.25)\end{array}$ & $\begin{array}{c}1 \\
(1.2)\end{array}$ & 0 & 0 & 2 \\
\hline Recycled & $\begin{array}{c}40 \\
(48.19)\end{array}$ & $\begin{array}{c}17 \\
(20.48)\end{array}$ & $\begin{array}{c}12 \\
(14.46)\end{array}$ & $\begin{array}{c}9 \\
(10.84)\end{array}$ & $\begin{array}{c}1 \\
(1.2)\end{array}$ & $\begin{array}{c}4 \\
(4.8)\end{array}$ & 0 & 0 & $\begin{array}{c}1 \\
(1.2)\end{array}$ & 1 \\
\hline Warehoused & $\begin{array}{c}21 \\
(25.30)\end{array}$ & $\begin{array}{c}27 \\
(32.5)\end{array}$ & $\begin{array}{c}21 \\
(25.30)\end{array}$ & $\begin{array}{c}2 \\
(2.4)\end{array}$ & $\begin{array}{c}2 \\
(2.4)\end{array}$ & $\begin{array}{c}10 \\
(12.05)\end{array}$ & 0 & 0 & $\begin{array}{c}1 \\
(1.2)\end{array}$ & 5 \\
\hline Thrown away & $\begin{array}{c}23 \\
(27.7)\end{array}$ & $\begin{array}{c}26 \\
(31.33) \\
\end{array}$ & $\begin{array}{c}20 \\
(24.09) \\
\end{array}$ & $\begin{array}{c}3 \\
(3.6) \\
\end{array}$ & $\begin{array}{c}2 \\
(2.4)\end{array}$ & $\begin{array}{c}9 \\
(10.84)\end{array}$ & 0 & 0 & $\begin{array}{c}1 \\
(1.2)\end{array}$ & 4 \\
\hline $\begin{array}{c}\text { Converted to other } \\
\text { products }\end{array}$ & $\begin{array}{c}26 \\
(31.33) \\
\end{array}$ & $\begin{array}{c}27 \\
(32.5)\end{array}$ & $\begin{array}{c}16 \\
(19.27) \\
\end{array}$ & $\begin{array}{c}5 \\
(6.02) \\
\end{array}$ & $\begin{array}{c}2 \\
(2.4) \\
\end{array}$ & $\begin{array}{c}7 \\
(8.43) \\
\end{array}$ & 0 & 0 & $\begin{array}{c}1 \\
(1.2) \\
\end{array}$ & 4 \\
\hline TOTAL & $\begin{array}{c}147 \\
(177.11) \\
\end{array}$ & $\begin{array}{c}122 \\
(146.99) \\
\end{array}$ & $\begin{array}{c}76 \\
(91.57) \\
\end{array}$ & $\begin{array}{c}21 \\
(25.3)\end{array}$ & $\begin{array}{c}8 \\
(9.6) \\
\end{array}$ & $\begin{array}{c}41 \\
(49.4) \\
\end{array}$ & $\begin{array}{c}1 \\
(1.2) \\
\end{array}$ & 0 & $\begin{array}{c}4 \\
(4.8) \\
\end{array}$ & \\
\hline
\end{tabular}

The school practiced recycling of non-biodegradable materials with 49 or $59.04 \%$ of the total responses, wherein 40 or $48.19 \%$ came from students, 9 or $10.84 \%$ from the faculty members, and no response from the school official. Other non-biodegradable waste was sent to recyclers with 37 or $44.58 \%$ from the students, 2 or $2.4 \%$ from the faculty members, the school official gave a positive response. Converted to other product with 26 or $31.32 \%$ coming from the students, 5 or $6.02 \%$ coming from the faculty members, and there was no response from the school official. Thrown away with 23 or $27.7 \%$ came from the students, 3 or $3.6 \%$ from the faculty members while the school official had no response. Warehouse with 21 or $25.3 \%$ coming from the students, 2 or $2.4 \%$ from the faculty members and there was no response from the school official. This means that the school still favored and practiced the recycling of non-biogradable wastes. 
Table 14: Responses of Faculty Members, School Official and Students on Disposing Chemical/Hazardous Waste

\begin{tabular}{|c|c|c|c|c|c|c|c|c|c|c|}
\hline \multirow[b]{3}{*}{ Ways/Practices } & \multicolumn{3}{|c|}{ Students } & \multicolumn{3}{|c|}{ Faculty Members } & \multicolumn{3}{|c|}{ School Official } & \multirow[b]{3}{*}{ RANK } \\
\hline & $\mathbf{P}$ & $\mathbf{N}$ & NR & $\mathbf{P}$ & $\mathbf{N}$ & NR & $\mathbf{P}$ & $\mathbf{N}$ & NR & \\
\hline & $\begin{array}{c}\mathbf{f} \\
(\%)\end{array}$ & $\begin{array}{c}\mathbf{f} \\
(\%) \\
\end{array}$ & $\begin{array}{c}\mathbf{f} \\
(\%)\end{array}$ & $\begin{array}{c}\mathbf{F} \\
(\%) \\
\end{array}$ & $\begin{array}{c}\mathbf{f} \\
(\%)\end{array}$ & $\begin{array}{c}\mathbf{f} \\
(\%)\end{array}$ & $\begin{array}{c}\mathbf{f} \\
(\%)\end{array}$ & $\begin{array}{c}\mathbf{f} \\
(\%) \\
\end{array}$ & $\begin{array}{c}\mathbf{f} \\
(\%) \\
\end{array}$ & \\
\hline Dispose of separately & $\begin{array}{c}41 \\
(49.4) \\
\end{array}$ & $\begin{array}{c}17 \\
(20.48) \\
\end{array}$ & $\begin{array}{c}11 \\
(13.25) \\
\end{array}$ & $\begin{array}{c}9 \\
(10.84) \\
\end{array}$ & $\begin{array}{c}1 \\
(1.2) \\
\end{array}$ & $\begin{array}{c}4 \\
(4.8) \\
\end{array}$ & $\begin{array}{c}1 \\
(1.2) \\
\end{array}$ & 0 & 0 & 1 \\
\hline $\begin{array}{c}\text { Segregated properly } \\
\text { before disposal }\end{array}$ & $\begin{array}{c}36 \\
(47.37) \\
\end{array}$ & $\begin{array}{c}23 \\
(27.7) \\
\end{array}$ & $\begin{array}{c}10 \\
(12.05)\end{array}$ & $\begin{array}{c}7 \\
(8.4) \\
\end{array}$ & $\begin{array}{c}1 \\
(1.2)\end{array}$ & $\begin{array}{c}6 \\
(7.23) \\
\end{array}$ & $\begin{array}{c}1 \\
(1.2)\end{array}$ & 0 & 0 & 2 \\
\hline $\begin{array}{c}\text { Rechargeable } \\
\text { batteries are } \\
\text { prepared over } \\
\text { disposal } \\
\end{array}$ & $\begin{array}{c}21 \\
(25.30)\end{array}$ & $\begin{array}{c}29 \\
(34.93)\end{array}$ & $\begin{array}{c}19 \\
(22.89)\end{array}$ & $\begin{array}{c}4 \\
(4.8)\end{array}$ & 0 & $\begin{array}{c}10 \\
(12.05)\end{array}$ & 0 & 0 & $\begin{array}{c}1 \\
(1.2)\end{array}$ & 4 \\
\hline $\begin{array}{c}\text { Toner and ribbon } \\
\text { cartridges are } \\
\text { bought }\end{array}$ & $\begin{array}{c}21 \\
(25.30)\end{array}$ & $\begin{array}{c}36 \\
(47.37)\end{array}$ & $\begin{array}{c}12 \\
(14.46)\end{array}$ & $\begin{array}{c}8 \\
(9.6)\end{array}$ & $\begin{array}{c}2 \\
(2.4)\end{array}$ & $\begin{array}{c}4 \\
(4.8)\end{array}$ & 0 & 0 & $\begin{array}{c}1 \\
(1.2)\end{array}$ & 3 \\
\hline TOTAL & $\begin{array}{c}119 \\
(143.97) \\
\end{array}$ & $\begin{array}{c}105 \\
(126.5) \\
\end{array}$ & $\begin{array}{c}52 \\
(62.65)\end{array}$ & $\begin{array}{c}28 \\
(33.73)\end{array}$ & $\begin{array}{c}4 \\
(4.8)\end{array}$ & $\begin{array}{c}24 \\
(28.9) \\
\end{array}$ & $\begin{array}{c}2 \\
(2.4)\end{array}$ & 0 & $\begin{array}{c}2 \\
(2.4) \\
\end{array}$ & \\
\hline
\end{tabular}

On the disposal of chemical/hazardous materials, separatedisposal is preferred by 41 or $49.4 \%$ of the students, 9 or $10.84 \%$ faculty members, the school official gave a positive response. Segregated properly before disposal with 36 or $43.37 \%$ coming from the students, 7 or $8.4 \%$ from the faculty members and the school official gave a positive response. Toner and ribbon cartridges were bought with 21 or $25.3 \%$ coming from the students, 8 or $9.6 \%$ from the faculty members, there is no response from the school official. Rechargeable batteries were preferred over disposal with 21 or $25.3 \%$ coming from the students, 4 or $4.8 \%$ from the faculty members, while the school official had no response. This means that the school was aware of disposing separately the hazardous waste from the usual/domestic wastes.

\subsection{Air Quality}

Table 15: Responses of Faculty Members, School Official, and Students on Air Quality Management

\begin{tabular}{|c|c|c|c|c|c|c|c|c|c|c|}
\hline \multirow[b]{3}{*}{ Practices } & \multicolumn{3}{|c|}{ Students } & \multicolumn{3}{|c|}{ Faculty Members } & \multicolumn{3}{|c|}{ School Official } & \multirow[b]{3}{*}{ RANK } \\
\hline & $\mathbf{P}$ & $\mathbf{N}$ & NR & $\mathbf{P}$ & $\mathbf{N}$ & NR & $\mathbf{P}$ & $\mathbf{N}$ & NR & \\
\hline & $\begin{array}{c}\mathbf{f} \\
(\%)\end{array}$ & $\begin{array}{c}\mathbf{f} \\
(\%)\end{array}$ & $\begin{array}{c}\text { f } \\
(\%)\end{array}$ & $\begin{array}{c}\mathbf{F} \\
(\%)\end{array}$ & $\begin{array}{c}\text { f } \\
(\%)\end{array}$ & $\begin{array}{c}\mathbf{f} \\
(\%)\end{array}$ & $\begin{array}{c}\text { f } \\
(\%)\end{array}$ & $\begin{array}{c}\text { f } \\
(\%)\end{array}$ & $\begin{array}{c}\mathbf{f} \\
(\%)\end{array}$ & \\
\hline $\begin{array}{c}\text { Smoking is } \\
\text { banned in } \\
\text { the entire } \\
\text { campus }\end{array}$ & $\begin{array}{c}6 \\
(7.23)\end{array}$ & $\begin{array}{c}13 \\
(15.66)\end{array}$ & $\begin{array}{c}50 \\
(60.24)\end{array}$ & $\begin{array}{c}3 \\
(3.6)\end{array}$ & 0 & $\begin{array}{c}11 \\
(13.25)\end{array}$ & $\begin{array}{c}1 \\
(1.2)\end{array}$ & 0 & 0 & 4 \\
\hline $\begin{array}{l}\text { Banned in all } \\
\text { closed area }\end{array}$ & $\begin{array}{c}15 \\
(18.2)\end{array}$ & $\begin{array}{c}32 \\
(38.55)\end{array}$ & $\begin{array}{c}22 \\
(26.5)\end{array}$ & $\begin{array}{c}3 \\
(3.6)\end{array}$ & $\begin{array}{c}2 \\
(2.4)\end{array}$ & $\begin{array}{c}9 \\
(10.84)\end{array}$ & 0 & 0 & $\begin{array}{c}1 \\
(1.2)\end{array}$ & 3 \\
\hline $\begin{array}{l}\text { Smoking is } \\
\text { allowed in } \\
\text { designated } \\
\text { areas }\end{array}$ & $\begin{array}{c}17 \\
(20.48)\end{array}$ & $\begin{array}{c}32 \\
(38.55)\end{array}$ & $\begin{array}{c}20 \\
(24.09)\end{array}$ & $\begin{array}{c}2 \\
(2.4)\end{array}$ & $\begin{array}{c}2 \\
(2.4)\end{array}$ & $\begin{array}{c}10 \\
(12.05)\end{array}$ & 0 & 0 & $\begin{array}{c}1 \\
(1.2)\end{array}$ & 2 \\
\hline $\begin{array}{c}\text { Smoke } \\
\text { belching }\end{array}$ & $\begin{array}{c}36 \\
(43.37)\end{array}$ & $\begin{array}{c}17 \\
(20.48)\end{array}$ & $\begin{array}{c}16 \\
(19.27)\end{array}$ & $\begin{array}{c}6 \\
(7.23)\end{array}$ & $\begin{array}{c}2 \\
(2.4)\end{array}$ & $\begin{array}{c}6 \\
(7.23)\end{array}$ & $\begin{array}{c}1 \\
(1.2)\end{array}$ & 0 & 0 & 1 \\
\hline
\end{tabular}




\begin{tabular}{|c|c|c|c|c|c|c|c|c|c|c|}
\hline $\begin{array}{c}\text { vehicles are } \\
\text { not allowed } \\
\text { inside the } \\
\text { campus }\end{array}$ & & & & & & & & & & \\
\hline TOTAL & 74 & 94 & 108 & 14 & 6 & 36 & 2 & & 2 & \\
$(89.16)$ & $(113.25)$ & $(130.12)$ & $(16.87)$ & $(7.23)$ & $(43.37)$ & $(2.4)$ & 0 & $(2.4)$ & \\
\hline
\end{tabular}

A majority of the respondents agreed that smoke belching vehicles were not allowed inside the campus. Ranked second was smoking is allowed in designated areas with 19 or $22.89 \%$ positive response wherein 17 or $20.48 \%$ coming from the students, 2 or $2.4 \%$ from the faculty members, and there was no response from the school official. Smoking is banned on the entire campus ranked the least with a total of 10 or $12.05 \%$ wherein 6 or $7.23 \%$ came from the students, 3 or $3.6 \%$ from the faculty members, the school official gave a positive response. Air quality management was also observed in the campus.

There was integration of environmental concerns/theme in the curriculum as part of enhancing environmental education among the students.

Science subject ranked the highest of about 75 positive responses or $90.36 \%$, wherein 62 or $74.7 \%$ from the students, 13 or $15.66 \%$ from the faculty members, the school official gave a positive response. English and Values were ranked second with 60 or $72.29 \%$ from the students, 12 or $14.45 \%$ from the faculty members. the school official gave a positive response. The third ranked subjects were Filipino and Mathematics of about 67 positive response or $80.7 \%$. The fourth ranked were Music, Health and Physical Education with 57 or $68.67 \%$ from the students, 12 or $14.45 \%$ from the faculty members, the school official gave a positive response. Last in the rank were Home Economics and Social Studies of about 61 positive response or $73.49 \%$. This indicates that integration of environmental themes/subjects were evident in most of their science subjects.

Table 16: Responses of Faculty Members, School Official, and Students on the Integration of Environmental Concerns/Themes in the Curriculum

\begin{tabular}{|c|c|c|c|c|c|c|c|c|c|c|}
\hline \multirow[b]{3}{*}{ Subjects } & \multicolumn{3}{|c|}{ Students } & \multicolumn{3}{|c|}{ Faculty Members } & \multicolumn{3}{|c|}{ School Official } & \multirow[b]{3}{*}{ RANK } \\
\hline & $\mathbf{P}$ & $\mathbf{N}$ & NR & $\mathbf{P}$ & $\mathbf{N}$ & NR & $\mathbf{P}$ & $\mathbf{N}$ & NR & \\
\hline & $\begin{array}{c}\mathbf{f} \\
(\%)\end{array}$ & $\begin{array}{c}f \\
(\%)\end{array}$ & $\begin{array}{c}f \\
(\%)\end{array}$ & $\begin{array}{l}\mathbf{F} \\
(\%)\end{array}$ & $\begin{array}{c}\mathbf{f} \\
(\%)\end{array}$ & $\begin{array}{c}\mathbf{f} \\
(\%)\end{array}$ & $\begin{array}{c} \\
(\%)\end{array}$ & $\begin{array}{c}\mathbf{f} \\
(\%)\end{array}$ & $\begin{array}{c}\mathbf{f} \\
(\%)\end{array}$ & \\
\hline English & $\begin{array}{c}60 \\
(72.29) \\
\end{array}$ & $\begin{array}{c}7 \\
(8.4)\end{array}$ & $\begin{array}{c}2 \\
(2.4) \\
\end{array}$ & $\begin{array}{c}12 \\
(14.45)\end{array}$ & 0 & $\begin{array}{c}2 \\
(2.4)\end{array}$ & 0 & 0 & $\begin{array}{c}1 \\
(1.2)\end{array}$ & 2 \\
\hline Filipino & $\begin{array}{c}56 \\
(67.47) \\
\end{array}$ & $\begin{array}{c}9 \\
(10.84)\end{array}$ & $\begin{array}{c}4 \\
(4.8) \\
\end{array}$ & $\begin{array}{c}11 \\
(13.25)\end{array}$ & 0 & $\begin{array}{c}3 \\
(3.6) \\
\end{array}$ & 0 & 0 & $\begin{array}{c}1 \\
(1.2) \\
\end{array}$ & 3 \\
\hline Social Studies & $\begin{array}{c}49 \\
(59.04)\end{array}$ & $\begin{array}{c}15 \\
(18.02)\end{array}$ & $\begin{array}{c}5 \\
(6.02)\end{array}$ & $\begin{array}{c}11 \\
(13.25)\end{array}$ & 0 & $\begin{array}{c}3 \\
(3.6)\end{array}$ & $\begin{array}{c}1 \\
(1.2)\end{array}$ & 0 & 0 & 5 \\
\hline $\begin{array}{l}\text { Music Health and } \\
\text { Physical Education }\end{array}$ & $\begin{array}{c}57 \\
(68.67)\end{array}$ & $\begin{array}{c}9 \\
(10.84)\end{array}$ & $\begin{array}{c}3 \\
(3.6)\end{array}$ & $\begin{array}{c}12 \\
(14.45)\end{array}$ & 0 & $\begin{array}{c}2 \\
(2.4)\end{array}$ & $\begin{array}{c}1 \\
(1.2)\end{array}$ & 0 & 0 & 4 \\
\hline Home Economics & $\begin{array}{c}49 \\
(59.04)\end{array}$ & $\begin{array}{c}13 \\
(15.66)\end{array}$ & $\begin{array}{c}7 \\
(8.4)\end{array}$ & $\begin{array}{c}11 \\
(13.25)\end{array}$ & 0 & $\begin{array}{c}3 \\
(3.6)\end{array}$ & $\begin{array}{c}1 \\
(1.2)\end{array}$ & 0 & 0 & 5 \\
\hline Mathematics & $\begin{array}{c}55 \\
(66.27)\end{array}$ & $\begin{array}{c}10 \\
(12.05)\end{array}$ & $\begin{array}{c}4 \\
(4.8)\end{array}$ & $\begin{array}{c}11 \\
(13.25)\end{array}$ & 0 & $\begin{array}{c}3 \\
(3.6)\end{array}$ & $\begin{array}{c}1 \\
(1.2)\end{array}$ & 0 & 0 & 3 \\
\hline Science & $\begin{array}{c}62 \\
(74.7)\end{array}$ & $\begin{array}{c}5 \\
(6.02)\end{array}$ & $\begin{array}{c}2 \\
(2.4)\end{array}$ & $\begin{array}{c}13 \\
(15.66)\end{array}$ & 0 & $\begin{array}{c}1 \\
(1.2)\end{array}$ & $\begin{array}{c}1 \\
(1.2)\end{array}$ & 0 & 0 & 1 \\
\hline
\end{tabular}


DOI: $10.5281 /$ zenodo.2619482

\begin{tabular}{|c|c|c|c|c|c|c|c|c|c|c|}
\hline Values & 59 & 8 & 2 & 12 & & 2 & 1 & & & \\
& $(71.08)$ & $(9.6)$ & $(2.4)$ & $(14.45)$ & 0 & $(2.4)$ & $(1.2)$ & 0 & 0 & 2 \\
\hline TOTAL & 447 & 76 & 29 & 93 & & 19 & 6 & 0 & 2 & \\
\hline
\end{tabular}

The data indicate that the environmental themes/concerns were integrated in the above-mentioned subjects. Teachers in Home Economics and Social Studies should endeavor to incorporate environmental themes in their subjects.

Table 19: Responses of Faculty Members, School Official, and Students on the Adequacy and Usage of Audio-Visual Materials regarding Environment

\begin{tabular}{|c|c|c|c|c|c|c|c|c|c|c|}
\hline \multirow[b]{3}{*}{ Practices } & \multicolumn{3}{|c|}{ Students } & \multicolumn{3}{|c|}{ Faculty Members } & \multicolumn{3}{|c|}{ School Official } & \multirow[b]{3}{*}{ RANK } \\
\hline & $\mathbf{P}$ & $\mathbf{N}$ & NR & $\mathbf{P}$ & $\mathbf{N}$ & NR & $\mathbf{P}$ & $\mathbf{N}$ & NR & \\
\hline & $\begin{array}{c}\mathbf{f} \\
(\%)\end{array}$ & $\begin{array}{c}\mathbf{f} \\
(\%)\end{array}$ & $\begin{array}{c}\mathbf{f} \\
(\%)\end{array}$ & $\begin{array}{c}\mathrm{F} \\
(\%)\end{array}$ & $\begin{array}{c}\mathbf{f} \\
(\%)\end{array}$ & $\begin{array}{c}\mathbf{f} \\
(\%)\end{array}$ & $\begin{array}{c}\mathbf{f} \\
(\%)\end{array}$ & $\begin{array}{c}\mathbf{f} \\
(\%)\end{array}$ & $\begin{array}{c}\mathbf{f} \\
(\%)\end{array}$ & \\
\hline $\begin{array}{l}\text { Student have easy to } \\
\text { access these resources }\end{array}$ & $\begin{array}{c}33 \\
(39.75) \\
\end{array}$ & $\begin{array}{c}15 \\
(18.07)\end{array}$ & $\begin{array}{c}51 \\
(61.4) \\
\end{array}$ & $\begin{array}{c}3 \\
(3.6)\end{array}$ & 0 & $\begin{array}{c}11 \\
(13.25)\end{array}$ & 0 & 0 & $\begin{array}{c}1 \\
(1.2)\end{array}$ & 2 \\
\hline $\begin{array}{c}\text { Students are } \\
\text { encouraged to make } \\
\text { use of these resources }\end{array}$ & $\begin{array}{c}31 \\
(37.35)\end{array}$ & $\begin{array}{c}18 \\
(21.69)\end{array}$ & $\begin{array}{c}20 \\
(24.09)\end{array}$ & $\begin{array}{c}7 \\
(8.4)\end{array}$ & 0 & $\begin{array}{c}7 \\
(8.4)\end{array}$ & 0 & 0 & $\begin{array}{c}1 \\
(1.2)\end{array}$ & 1 \\
\hline TOTAL & $\begin{array}{c}64 \\
(77.1)\end{array}$ & $\begin{array}{c}33 \\
(39.75)\end{array}$ & $\begin{array}{c}71 \\
(81.5)\end{array}$ & $\begin{array}{c}10 \\
(12.05)\end{array}$ & 0 & $\begin{array}{c}18 \\
(21.69)\end{array}$ & 0 & 0 & $\begin{array}{c}2 \\
(2.4)\end{array}$ & \\
\hline
\end{tabular}

The adequacy and use of audio-visual materials on environment were encouraged to make use of the audio-video materials regarding the environment with 36 positive respondents' response. Furthermore, the students had positive response on the accessibility of these resources. This means that the school had relatively adequate audio-visual materials regarding the environment and that these were accessible for use by the students.

\section{Conclusions}

Based on the findings of the study, the following conclusions were drawn ; the operational standards and proper codes implemented by the school includes the following: the building and grounds are designed to make use of natural ventilation and natural lighting, Fluorescent lamps are commonly used as sources of light, Water source from the local waterworks system, Office machines/equipment are turned off when not in use, and electric fans are used whenever possible as compared to usage of air conditioners, White paper is the most preferred kind of paper used in school, Plastic utensils are most discouraged materials in the canteen and Waste segregation is practiced in school and containers for biodegradable wastes are provided in the entire campus. The environmental education is part of the academic development as manifested in the Environmental themes/concerns are integrated in the curriculum, particularly in Science, English, Values Education, Filipino, Mathematics, Music, Health and Physical Education, but with lesser integration in Home Economics and Social Studies.

\section{Recommendations}

The following recommendations are presented, review and/or restructure the mission of the school and incorporate an environmental thrust so that the school would further improve, adopt an 
environmental policy and regularly attend/conduct seminars regarding solid waste management and related environmental issues/themes.

\section{References}

[1] Anes, Myrna., and Sergio J. Lee. 2008. Lecture Notes in Environmental Science: the Economy of Nature and Ecology of Man. C and E Publishing Inc., 839 EDSA, South Triangle, Quezon City.

[2] Barnes, David. British Antarctic Survey. Philosophical Transactions of The Royal Society B Scientific Journal. 2009

[3] Broto, Antonio S. 2006. Statistics Made Simple, 2nd Edition. National bookstore, Quad Alpha Centrum Bldg, 125 H. Pioneer Street, Mandaluyong City.

[4] Cohen, M.R. 1973. "Environmental Information Versus Environmental Attitude" The Journal of Environmental Education

[5] Cunningham, William P. And Mary-an Cunningham. 2003. Environmental Science, A Global Concern, 7th ed. St. Cloud State University.

[6] Environmental Education Guide, 1999, Asian Development Bank: TA.No.1385 phi, CPSCTE and MEMS Inc.

[7] Environmental laws in the Philippines, 3rd edition. 2005 CBSI Editorial Staff, Central Bank Supply, Inc., Central Print on Demand, 927 Quezon Avenue, Quezon City Philippines.

[8] Guzman, Roger S. and Ruth Z. Guzman, 2000. Environmental Education for Sustainable Development.

[9] Leslie, R.P. Capturing the daylight dividend in buildings: why and how? Building and Environment Volume 38, Issue 2, February 2003, Pages 381-385

[10] Meneses, Gonzalo Díaz. Recycling as an Object of Study for Behavioural Sciences. December 30, 2015. Advances in Recycling \& Waste Management.

[11] Mina Fe C., (undated) "Zero Waste Management Program and its Implication to Society." The Modern Teachers, Vol. XLVIII. No.1

[12] Pearce, David W. And Turner, Kerry R. 1990. Economics of Natural Resources and the Environment. Publish in Great Britain.

[13] Pearson, Brian, Barry F.P Little and Marcus Jane Brierly. 1992. Using Environmental Management Systems to Improve Profits. Cower Law International, Sterling House 66 Wilton Road, London United Kingdom

[14] Saigo Barbara and William Cunningham. 1995. Environmental Science: A Global Concern, 3rd edition. Wm.C Brown Publishers, Southeastern Louisiana University.

[15] Sinha, Shradha, Manishe shukla and Ranjan Shukla. 2005. A Textbook of Environments. Virender Kumar Arya. AITBS Publishers and Distributors, j-5/6 Krishan Nagar, New elhi- 11051(INDIA).

[16] Ramsey, J.M. and Hungerford H.R. 1989, "The Effects of Issue Investigated and Action Training on Environmental Education" North American Association For Environmental Education. 23(2), 34-35.

[17] Rodriguez, Felix L. (1995) Lecture Presented at the Second ASEAN Conference on Environmental Education." Philippines Geographical Journal; pg12-19.

[18] Sagay,Teresita C. (1997). "Greening our Environment." The Modern Teacher, Vol. XIV, No.10. 